\title{
PHACOEMULSIFICATION DURING THE LEARNING CURVE: RISK/BENEFIT ANALYSIS
}

\author{
H. C. SEWARD, R. DALTON and A. DAVIS \\ Croydon
}

\begin{abstract}
SUMMARY
To assess prospectively the complications and outcomes in a single-surgeon series during the phacoemulsification learning curve a prospective analysis of 400 phacoemulsification procedures in 358 patients was carried out. The main outcome measures were capsular tear, vitreous and nuclear loss, best corrected post-operative visual acuity and refractive cylinder. Capsular tears occurred in 6.3\% of phacoemulsification procedures, with vitreous loss in $1.5 \%$. One nucleus was lost into the vitreous. Best corrected visual acuity, including pre-existing pathology, was $6 / 9$ or better in $83.6 \%$ of eyes. There was 1 dioptre or less change of astigmatism from pre-operative to 6 weeks in $91 \%$ of eyes. It is concluded that phacoemulsification with careful patient selection can be a safe procedure even during the learning curve.
\end{abstract}

Phacoemulsification was first described by Kelman in 1967. ${ }^{1}$ The original aim of phacoemulsification was to reduce the convalescent period by reducing the then $180^{\circ}$ incision size for routine intracapsular cataract surgery (ICCE). The technique was not popularised until the 1980s when the advent of viscoelastic materials and improved instrumentation together with capsulorhexis and phacoemulsification of the lens in the posterior chamber provided much greater corneal protection. ${ }^{2}$ This proved to be the impetus necessary, such that now phacoemulsification is the preferred method of cataract surgery in the United States, with $52 \%$ of members of the American Cataract and Refractive Surgery Society preferring phacoemulsification to planned extracapsular cataract extraction (ECCE). ${ }^{3}$ ECCE with posterior chamber lens implantation is the most popular method of cataract surgery in the United Kingdom at present, with a proven track record of success. ${ }^{4}$ Hodgkins et al. ${ }^{5}$ in a recent UK survey found that only $2 \%$ of surgeons preferred phacoemulsification. Steele ${ }^{6}$ in a recent editorial in the British Journal of Ophthalmology predicts the emergence of a different picture at the next survey.

Correspondence to: H. C. Seward, Croydon Eye Unit, 33 Mayday Road, Thornton Heath, Surrey CR7 7XN, UK.
However, many surgeons are unwilling to change to a new technique which is thought to have a very high complication rate during the learning curve. There are few publication $\mathrm{s}^{7-9}$ documenting these complications to advise the surgeon contemplating the transition to phacoemulsification, and none from the United Kingdom. The aim of this prospective study of 400 eyes undergoing phacoemulsification (phaco) is to document the complications and outcomes during the phaco learning curve by an experienced surgeon.

\section{PATIENTS AND METHODS}

\section{Patients}

This prospective study compares data obtained on two groups of patients all of whom were operated on by a single consultant surgeon. The study population is divided into two groups to assess the effect of increasing surgical experience on outcome.

The first group (phaco 1) comprised 200 eyes in 174 patients which were the initial 200 phaco cases performed by the surgeon. These eyes were assessed as suitable for phaco by a learning surgeon. Collection of data for this group took place between May 1989 and November 1990 and represented $35 \%$ of all cataract surgery performed by the surgeon during this period.

The second group (phaco 2) comprised 200 eyes in 184 patients having phacoemulsification between November 1990 and September 1991, representing 64\% of all cataract surgery performed by the surgeon during this period.

\section{Surgical Technique}

Phacoemulsification was performed under local anaesthesia in 146 (73\%) cases in the phaco 1 group, and in 158 (79\%) cases in the phaco 2 group.

The phacoemulsification incision site was anterior scleral in the first 100 cases and moved posteriorly $(2 \mathrm{~mm}$ from the limbus) thereafter. The incision size varied from $3 \mathrm{~mm}$, when no implant was used, to $7 \mathrm{~mm}$ for a bifocal implant. The posterior chamber lens used had a $6 \mathrm{~mm}$ 
optic; the small-incision lens a $5 \times 6 \mathrm{~mm}$ optic. Viscoelastic material was routinely used. In the phaco 1 group $60 \%$ of eyes had a 'can-opener' capsulotomy and a Maloney three-step phaco procedure. ${ }^{10}$ Capsulorhexis and in-thebag phacoemulsification was performed in $40 \%$. In the phaco 2 group capsulorhexis with in-the-bag phacoemulsification was the method used in $70 \%$ of eyes, the other $30 \%$ having capsulotomy and a Maloney three-step procedure.

Wound closure was performed with a 10/0 nylon suture in bootlace configuration. Post-operative subconjunctival betamethasone and gentamicin were administered in both groups. The phacoemulsification in the phaco 1 group was entirely performed with a machine using a venturi pump system. This was the system also predominantly used in the phaco 2 group, although a machine with a diaphragmatic pump was used in 40 cases.

\section{Data Collection}

Prospective collection of data was made on a standard form for all patients in both groups. Age, sex, and known ocular and systemic disease were recorded for all patients. Pre-operative refractive cylinder and lens type (and therefore incision size) were noted in both groups. Intra-operative complications, including posterior capsule rupture and vitreous loss, were noted. No attempt was made intraoperatively to manipulate astigmatism. Endothelial cell counting facilities were not available.

Refraction was performed on all patients at 6 weeks by an optometrist in the department, to eliminate potential bias from refraction by the surgeon. (Thirteen patients did

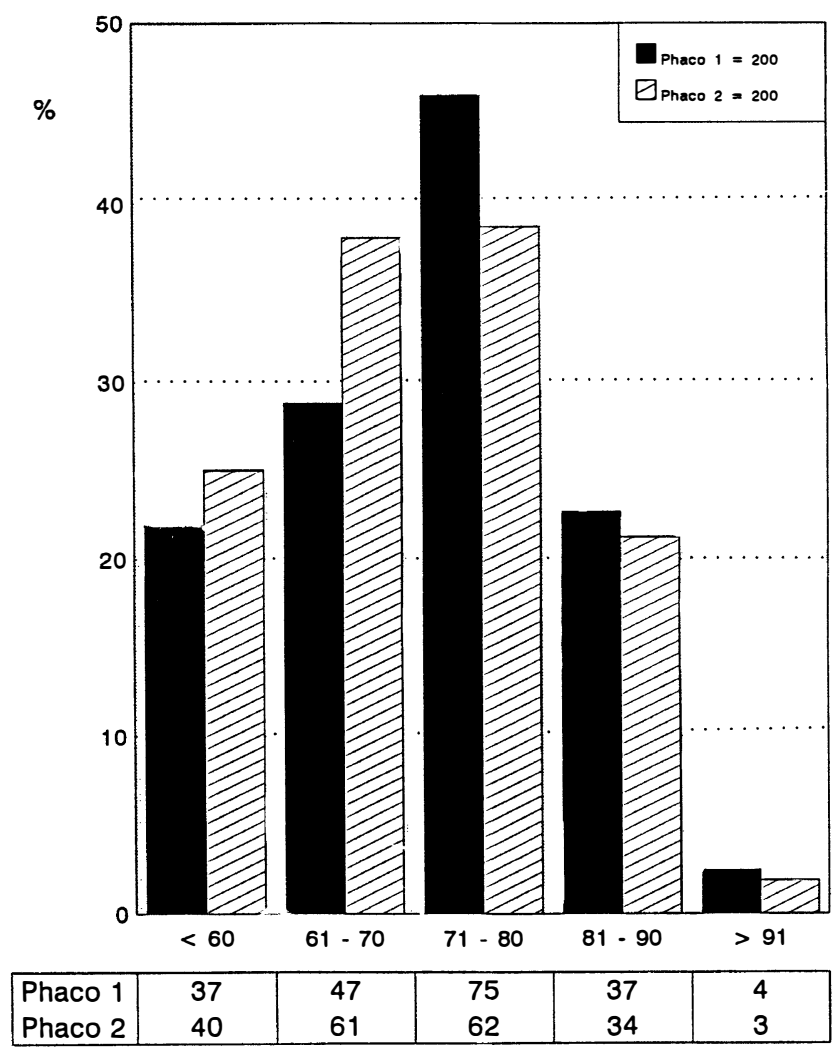

Fig. 1. Age distribution of patients. not attend for 6-week refraction.) On a subgroup of the patients (69 and 56 in the phaco 1 and 2 groups respectively) refraction was also performed at 6 months. At each refraction best corrected visual acuity, final sphere and cylinder were measured. The amount of astigmatic cylinder was measured by refractive and not keratometric methods. The reason for a final corrected visual acuity of $6 / 12$ or less was also noted in both groups of patients. A diagnosis of cystoid macular oedema was made clinically; fluorescein angiography was not performed.

\section{Data Analysis}

Tables used for statistical analysis were produced using the SPSS data entry and SPSS/PC systems. Illustrations were produced on the Harvard Graphics Version III. The Pearson chi-squared test was used to determine the different distributions of categorical variables between the groups, and a factor was determined as being statistically significant when $p \leqslant 0.05$.

Analysis of the cylinder was subsequently performed using the 'simple subtraction' method without regard to axis. $^{12}$

\section{RESULTS}

\section{Pre-operative Results}

The age distribution of the patients is shown in Fig. 1 (Pearson chi-squared $=3.43$, degrees of freedom $(\mathrm{df})=4, p=$ 0.49 ). It can be seen that patients in the phaco 2 group are younger. The sex distribution is similar in both groups (phaco 1, 72.5\% female; phaco 2, 64.5\% female). As would be expected there is an overall preponderance of women in the study (67\%).

Details of pre-existing ocular disease are shown in Table I. The distribution of age-related macular degeneration shows no significant difference between the two groups. Under the category 'other' were included corneal disease, previous retinal detachment, diabetic retinopathy and myopic degeneration. An established diagnosis of diabetes mellitus was present in $33(16.5 \%)$ of the phaco 1 group and $24(12.5 \%)$ of the phaco 2 group.

\section{Intra-operative Results}

Vitreous loss, capsular tear and nuclear loss were considered to be the main intra-operative complications. There were no significant differences in these complications between the two groups (Table II): capsular tear was found in $7.5 \%$ of the phaco 1 group and $5.0 \%$ of the phaco 2 group, while vitreous loss rate was $2.0 \%$ for phaco 1 and

Table I. Pre-existing ocular disease

\begin{tabular}{lcc}
\hline & $\begin{array}{l}\text { Phaco 1 } \\
(n=200)\end{array}$ & $\begin{array}{l}\text { Phaco 2 } \\
(n=200)\end{array}$ \\
\hline ARMD & $14(7.0 \%)$ & $13 \quad(6.5 \%)$ \\
Glaucoma & $13(6.5 \%)$ & $33(1.5 \%)$ \\
Other & $26(13.0 \%)$ & $24(12.0 \%)$ \\
\hline
\end{tabular}

Pearson chi-squared $=4.64,2 \mathrm{df}, p=0.982$.

ARMD, age-related macular disease. 
Table II. Surgical complications

\begin{tabular}{|c|c|c|}
\hline & $\begin{array}{l}\text { Phaco } 1 \\
(n=200)\end{array}$ & $\begin{array}{l}\text { Phaco } 2 \\
(n=200)\end{array}$ \\
\hline Capsular tear & $15 \quad(7.5 \%)$ & $10 \quad(5.0 \%)$ \\
\hline Vitreous loss & $4 \quad(2.0 \%)$ & $(1.0 \%)$ \\
\hline Nuclear loss & $1 \quad(0.5 \%)$ & 0 \\
\hline
\end{tabular}

Pearson chi-squared $=0.09,1 \mathrm{df}, p=0.763$.

$1.0 \%$ for phaco 2 . One nucleus was lost into the vitreous in the phaco 1 group.

The type of lens used can be seen in Table III. The use of anterior chamber lenses was lower (1.0\%) in the phaco 2 group. Small-incision lenses were used in $13.5 \%$ of eyes in the phaco 1 group compared with $44.0 \%$ in the phaco 2 group; posterior chamber lenses were used in $73.0 \%$ of the phaco 1 group and $49.5 \%$ of the phaco 2 group. It can be seen that there is a highly significant difference in the distribution of lens types used between the two groups.

\section{Post-operative Results}

The best corrected visual acuity at 6 weeks following surgery is shown in Fig. 2 (Pearson chi-squared $=6.4, \mathrm{df}=5$, $p=0.27$ ). There was no significant difference in visual outcome between the two groups. The causes of vision of $6 / 12$ or less post-operatively are given in Table IV. These showed no significant difference between the two groups.

The refractive cylinder results 6 weeks following surgery are shown in Fig. 3 (Pearson chi-squared $=3.38, \mathrm{df}=$ $4, p=0.5$ ). There was no significant difference in astigmatism between the phaco 1 and phaco 2 groups: $53.9 \%$ of eyes in the phaco 2 group had $\leqslant 1$ dioptre of cylinder, compared with $56.3 \%$ in the phaco 1 group.

The change in cylinder from pre-operative to 6 weeks post-operative, using the simple subtraction method, is shown in Fig. 4 (Pearson chi-squared $=7.02, \mathrm{df}=7, p=$ 0.43 ). It can be seen that $47.1 \%$ and $56.5 \%$ of the phaco 1 and 2 groups respectively had no change in cylinder at 6 weeks. In the phaco 1 group $90.5 \%$ and in the phaco 2 group $91.3 \%$ were within 1 dioptre of their pre-operative cylinder at 6 weeks.

Refraction at 6 months was performed on 69 eyes in the phaco 1 group and 56 eyes in the phaco 2 group. There was $\leqslant 1$ dioptre change of cylinder from pre-operative to 6 months in $85.5 \%$ of the phaco 1 group and $96.4 \%$ of the phaco 2 group.

Iris prolapse occurred in $2(1.0 \%)$ of the phaco 1 group and none of the phaco 2 group. The follow-up range in months was 1-25 for the phaco 1 group and 1-14 for the

Table III. Type of lens implant

\begin{tabular}{|c|c|c|}
\hline & $\begin{array}{l}\text { Phaco 1 } \\
(n=200)\end{array}$ & $\begin{array}{l}\text { Phaco } 2 \\
(n=200)\end{array}$ \\
\hline Posterior chamber & $146(73.0 \%)$ & $99(49.5 \%)$ \\
\hline Multifocal & $22(11.0 \%)$ & $11(5.5 \%)$ \\
\hline Small-incision & $27(13.5 \%)$ & $88(44.0 \%)$ \\
\hline Anterior chamber & $4 \quad(2.0 \%)$ & $2(1.0 \%)$ \\
\hline No lens & $1 \quad(0.5 \%)$ & 0 \\
\hline
\end{tabular}

Pearson chi-squared $=45.70,3 \mathrm{df}, p=<0.0001$. phaco 2 group. The mode follow-up time was 6 months for both groups.

\section{DISCUSSION}

There are few studies ${ }^{7-9}$ which have attempted to quantify the complications during the phacoemulsification learning curve and most are retrospective. Allinson ${ }^{8}$ and Cruz ${ }^{9}$ document complications occurring whilst surgeons in training are learning phacoemulsification, whilst Peder$\operatorname{sen}^{7}$ is an experienced cataract surgeon.

We have endeavoured to assess prospectively the level of these complications. The design of the study is open to criticism in that patients were assigned to phacoemulsification on the basis of suitability by a learning phaco surgeon and endothelial cell counts were not performed. Further, two different phaco techniques were used, i.e. capsulotomy with the Maloney three-step technique and capsulorhexis with in-the-bag phacoemulsification. ${ }^{10}$

Analysis of the surgical complications shows no significant differences in capsular tear rate between the 2 groups. The capsular tear rate of the phaco 1 group (7.5\%) compared with that of Kershner, ${ }^{11}$ who had an $8 \%$ capsular tear rate in 1,000 phacoemulsification procedures using a 'canopener' capsulotomy. However, it is higher than Pedersen's ${ }^{7}$ rate of $4.9 \%$. The capsular tear rate of $5.0 \%$ in the phaco 2 group compares with the $4.0 \%$ capsular tear rate of Kershner ${ }^{11}$ in 1,000 cases of phaco with capsulorhexis. Cruz et al. ${ }^{9}$ found a capsular tear rate of $9.9 \%$. Thus it appears that the capsular tear rate decreases with a combination of increasing surgical experience and the use of capsulorhexis.

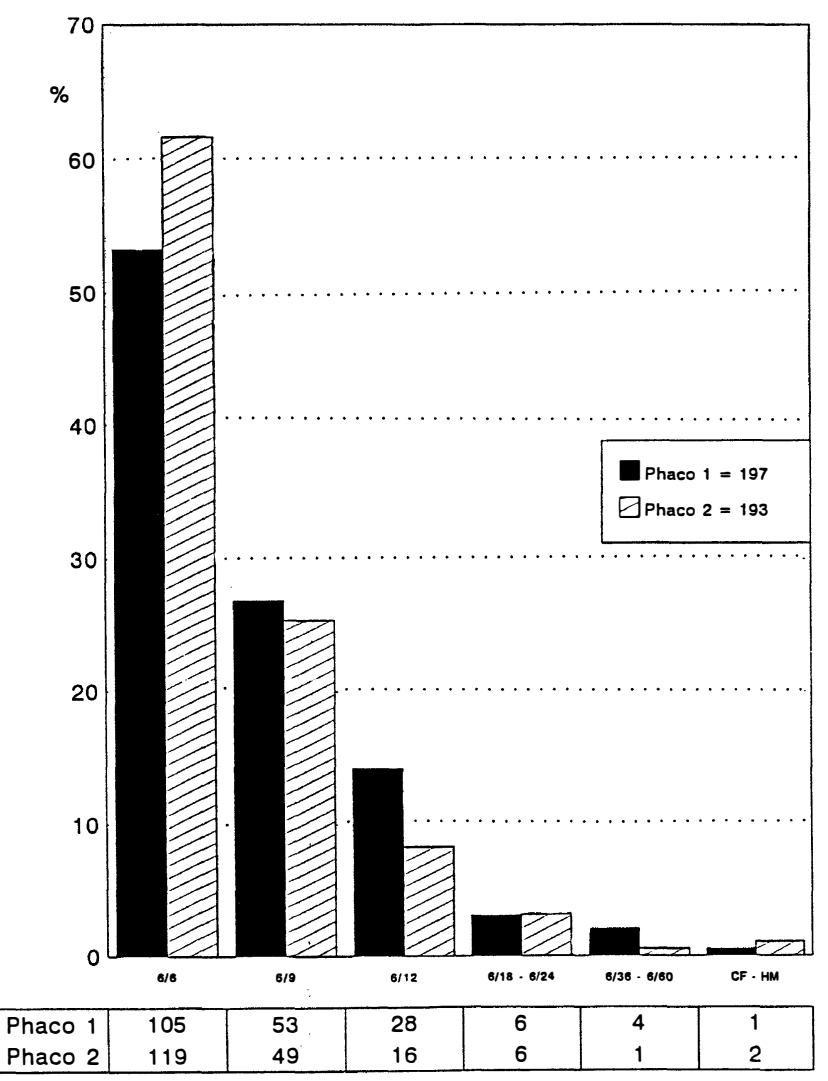

Fig. 2. Best corrected visual acuity at 6 weeks. 
Table IV. Reasons for visual acuity $\leqslant 6 / 12$

\begin{tabular}{lcccc}
\hline & $\begin{array}{l}\text { Phaco 1 } \\
(n=197)\end{array}$ & \multicolumn{2}{c}{$\begin{array}{l}\text { Phaco 2 } \\
(n=193)\end{array}$} \\
\hline ARMD & 9 & $(4.6 \%)$ & 8 & $(4.1 \%)$ \\
Glaucoma & 3 & $(1.5 \%)$ & 2 & $(1.0 \%)$ \\
CMO & $7(3.6 \%)$ & 4 & $(2.1 \%)$ \\
Other & $20(10.2 \%)$ & 10 & $(5.2 \%)$ \\
\hline
\end{tabular}

Pearson chi-squared $=0.66,3 \mathrm{df}, p=0.883$.

ARMD, age-related macular disease; CMO, cystoid macular oedema.

The vitreous loss rate in the phaco 1 group was $2 \%$. This compares with the $2.1 \%$ vitreous loss rate in Pedersen's series $^{7}$ but is considerably lower than the $14.7 \%$ in Allinson's series. ${ }^{8}$ The most dreaded complication of phaco, namely loss of the nucleus into the vitreous, occurred once in all 400 phacos. The literature contains few references to nuclear loss rate in phacoemulsification.

The use of anterior chamber lenses is always a good indication of surgical complications. Their use was higher in the phaco 1 group than in the phaco 2 group. The increasing use of capsulorhexis explains the increased use of small-incision lenses.

Visual results varied in the two groups, with $87 \%$ of the phaco 2 group seeing $6 / 9$ or better at 6 weeks compared with only $80.0 \%$ of the phaco 1 group. This difference was not significant. The visual results correspond well to reported series, ${ }^{5,7}$ which confirms that visual outcome need not be jeopardised during the phaco learning curve.

There was 1 patient in the phaco 1 group who had no perception of light 6 months following surgery. In this

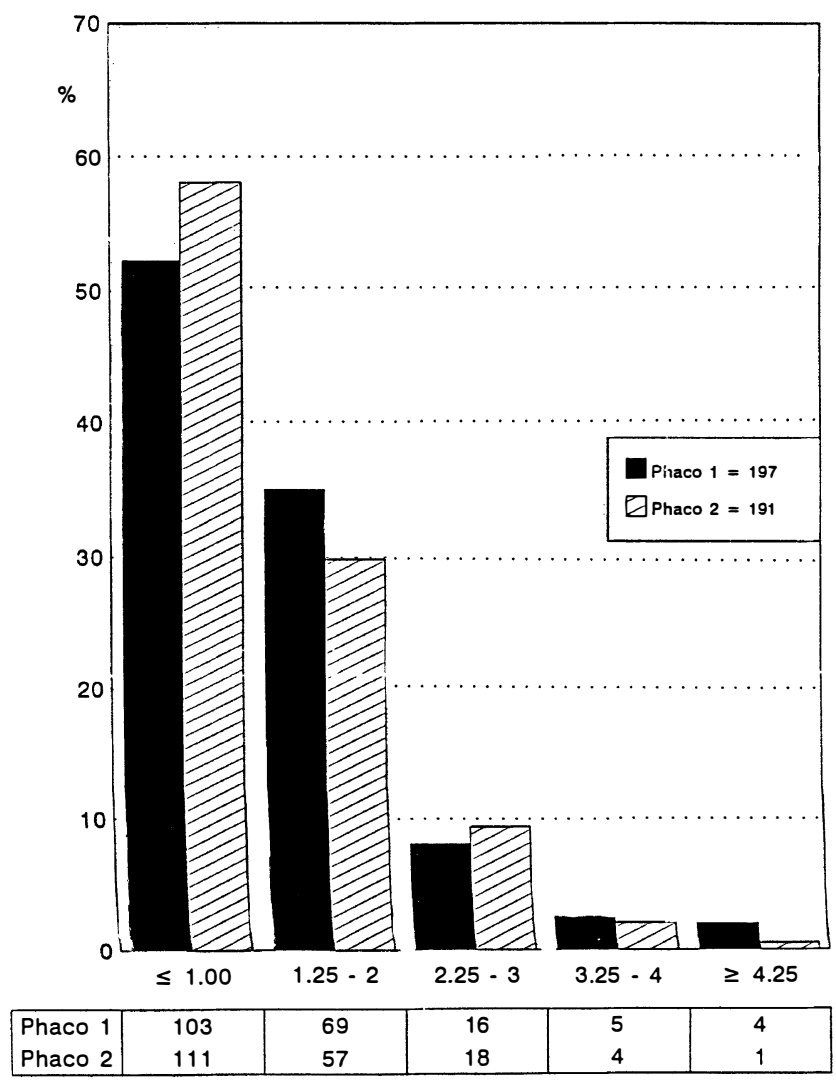

Fig. 3. Refractive cylinder at 6 weeks. patient the nucleus was lost into the vitreous at surgery. The patient later underwent vitrectomy, but developed a retinal detachment which did not respond successfully to treatment.

Astigmatism was measured in this study using refractive cylinder, which is less accurate than keratometric cylinder. It was, however, a single-surgeon series and astigmatism was not measured by the surgeon. Astigmatism at 6 weeks was similar in both groups.

Steinert et al $^{12}$ have summarised the advantages and disadvantages of three methods of calculating change in cylinder. Their paper shows that the three methods - the simple subtraction method, the axis induced cylinder method of Cravy, ${ }^{13}$ and the vector analysis method of Jaffe and Clayman $^{14}$ - provide similar results. We have measured change in cylinder using the simple subtraction method which ignores axis change (a shift of 1 dioptre with-the-rule to 1 dioptre against-the-rule is calculated as zero shift). Using this method to compare pre-operative, 6 week and 6 month changes in cylinder we found that almost 50\% are unchanged and that over $85 \%$ in each group have a difference of only 1 dioptre. This further supports the claim that phacoemulsification is an astigmatically neutral procedure. ${ }^{12.15}$

In conclusion, this paper documents the low complication rate during the learning curve of phaco for an experienced cataract surgeon. We would like to emphasise the importance of careful patient selection as discussed by Maloney. ${ }^{10}$ We attribute our low complication rate to

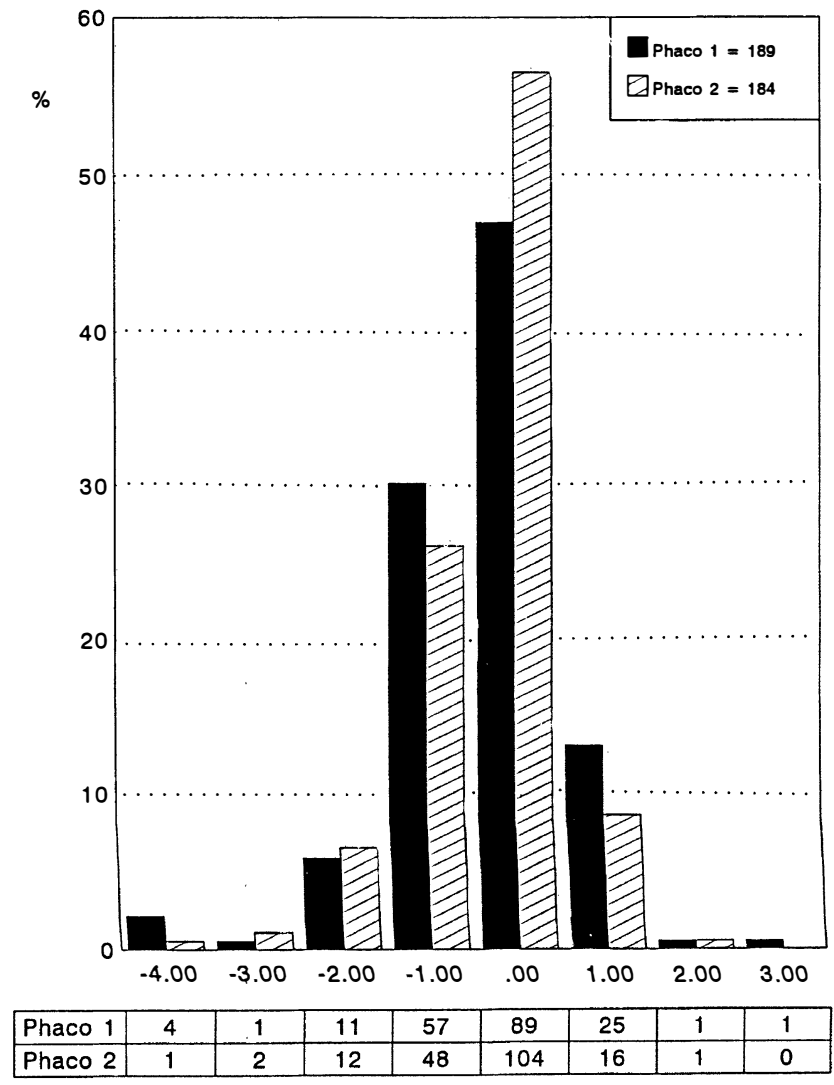

Fig. 4. Change in cylinder from pre-operative to 6 weeks post-operative. 
initial selection of soft cataracts, with more difficult cases being included only with increasing surgical experience. This is verified by the fact that the phaco 1 group represents $35 \%$ of all cataract extractions performed by the surgeon and the phaco 2 group 64\%. It is hoped that this prospective study will provide some basis for expectation regarding both complications and outcome for experienced cataract surgeons converting to phacoemulsification.

We would like to thank the Medical Statistic Department of Southampton Medical School, in particular Mr. S. Julius, for their invaluable help with the statistical analysis and illustrations and Ms. Amanda Hobbs for her secretarial assistance.

Key words: Astigmatism, Cataract Surgery, Complications, Learning curve, Phacoemulsification, Visual outcome.

\section{REFERENCES}

1. Kelman CD. Phacoemulsification and aspiration: a new technique of cataract extraction. Am J Ophthalmol 1967;64: 23-5.

2. Holmberg S, Philipson BT. Sodium hyaluronate in cataract surgery. VI. Report on use of Healon in extracapsular cataract extraction with phacoemulsification. Ophthalmology 1984;91:53-9.

3. Leaming DV. Practice styles and preferences of ASCRS members: 1990 survey. J Cataract Refract Surg 1991;17: 495-502.

4. Acheson JF, McHugh JD, Falcon MG. Changing patterns of early complications in cataract surgery with new techniques: a surgical audit. Br J Ophthalmol 1988;72:481-4.
5. Hodgkins PR, Luff AJ, Morrell AJ, Teye Botchway L, Featherston TJ, Fielder AR. Current practice of cataract extraction and anaesthesia. Br J Ophthalmol 1992;76: 323-6.

6. Steele AD McG. Cataract management [editorial]. Br J Ophthalmol 1992;76:321.

7. Pedersen O. Phacoemulsification and intraocular lens implantation in patients with cataract. Acta Ophthalmol (Copenh) 1990;68:59-64.

8. Allinson RW, Metrikin D, Fante R. Incidence of vitreous loss among third year residents performing phacoemulsification. Ophthalmology 1992;99:726-30.

9. Cruz OA, Wallace GW, Gay LA, Matoba AY, Koch DD. Visual results and complications of phacoemulsification with intraocular lens implantation performed by ophthalmology residents. Ophthalmology 1992;99:448-52.

10. Maloney WF, Grindle L. Textbook of phacoemulsification. California: Lapenda Publishers, 1988.

11. Kershner RM. Embryology, anatomy and needle capsulotomy. In: Koch PS, Davison JA, editors. Textbook of advanced phacoemulsification techniques. New Jersey: Slack International, 1991:35-48.

12. Steinert RF, Brint SF, White SM, Fine IH. Astigmatism after small incision cataract surgery. Ophthalmology 1991;98: 417-23.

13. Cravy TV. Calculation of the change in corneal astigmatism following cataract extraction. Ophthalmic Surg 1979;10: 38-49.

14. Jaffe NS, Clayman HM. The pathophysiology of corneal astigmatism after cataract extraction. Trans Am Acad Ophthalmol Otolaryngol 1975;79:OP615-30.

15. Palin SL. Comparison of induced astigmatism with phacoemulsification and extracapsular cataract extraction. J Cataract Refract Surg 1987;13:274-8. 